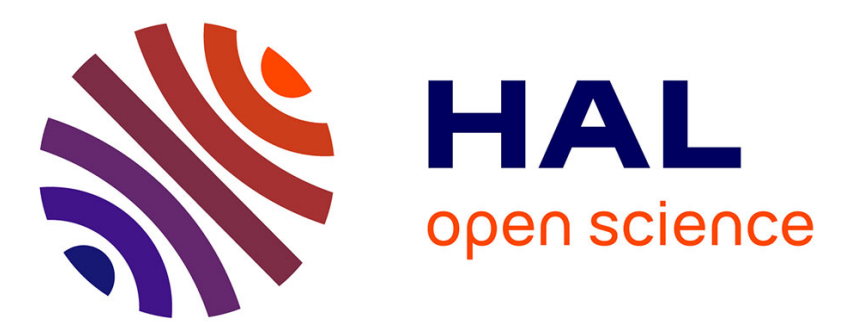

\title{
Nuclear magnetic relaxation dispersion investigations of water retention mechanism by cellulose ethers in mortars
}

Laetitia Patural, Jean-Pierre Korb, Alexandre Govin, Philippe Grosseau, Bertrand Ruot, Olivier Devès

\section{- To cite this version:}

Laetitia Patural, Jean-Pierre Korb, Alexandre Govin, Philippe Grosseau, Bertrand Ruot, et al.. Nuclear magnetic relaxation dispersion investigations of water retention mechanism by cellulose ethers in mortars. Cement and Concrete Research, 2012, 42 (10), pp.1371-1378. 10.1016/j.cemconres.2012.06.002 . hal-00762106

\section{HAL Id: hal-00762106 \\ https://hal.science/hal-00762106}

Submitted on 30 Jan 2013

HAL is a multi-disciplinary open access archive for the deposit and dissemination of scientific research documents, whether they are published or not. The documents may come from teaching and research institutions in France or abroad, or from public or private research centers.
L'archive ouverte pluridisciplinaire HAL, est destinée au dépôt et à la diffusion de documents scientifiques de niveau recherche, publiés ou non, émanant des établissements d'enseignement et de recherche français ou étrangers, des laboratoires publics ou privés. 
Nuclear magnetic relaxation dispersion investigations of water retention mechanism by cellulose ethers in mortars

4 Laetitia Patural ${ }^{(1)}$, Jean-Pierre Korb $^{(2)^{*}}$, Alexandre Govin $^{(1)}$, Philippe

5 Grosseau ${ }^{(1)}$, Bertrand RuOT $^{(3)}$, OLIVIER DEVÈS $^{(3)}$

6

(1) École Nationale Supérieure des Mines de Saint-Étienne ; Centre SPIN, LPMG FRE 3312 158 Cours Fauriel, 42023 Saint-Étienne Cedex 2, France

(2) Physique de la Matière Condensée, Ecole Polytechnique-CNRS, Route de Saclay, 91128 Palaiseau Cedex, France

(3) Université Paris-Est, Centre Scientifique et Technique du Bâtiment; Département Enveloppe et Revêtements / Division Enduits, Mortiers et Colles, 84 avenue Jean Jaurès,

$20 *$ Corresponding author: jean-pierre.korb@polytechnique.fr 


\section{ABSTRACT}

23

24

25

We show how nuclear magnetic spin-lattice relaxation dispersion of protons-water (NMRD) can be used to elucidate the effect of cellulose ethers on water retention and hydration delay of freshly-mixed white cement pastes. NMRD is useful to determine the surface diffusion coefficient of water, the specific area and the hydration kinetics of the cement-based material. In spite of modifications of the solution's viscosity, we show that the cellulosic derivatives do not modify the surface diffusion coefficient of water. Thus, the mobility of water present inside the medium is not affected by the presence of polymer. However, these admixtures modify significantly the surface fraction of mobile water molecules transiently present at solid surfaces. This quantity measured, for the first time, for all admixed cement pastes is thus relevant to explain the water retention mechanism.

\section{(1)}

KEYWORDS: cellulose ether, cement paste, NMR relaxometry, surface diffusion coefficient, water retention. 0 1 


\section{Introduction}

Cellulose ethers $(C E)$ are well known water-soluble semi-synthetic polymers derived from cellulose, the most abundant polysaccharide in nature. This polymer is build up from 1,4anhydroglucose units linked through $\beta-1,4$ glycoside bonds. Among the various derivatives of this polymer, hydroxypropyl methyl cellulose (HPMC, Fig. 1a), hydroxyethyl methyl cellulose (HEMC, Fig. 1b) and hydroxyethyl cellulose (HEC, Fig. 1c) are extensively used in the formulation of various industrial products encountered in food stuffs, pharmaceuticals and building materials. For instance, the presence of cellulose ethers $(C E)$ in mortars enhances drastically the water-retention [1]. Some experimental devices have been designed to evidence such water retention on a macroscopic lengthscale (Fig. 2). This specific property of $C E$ is crucial to achieve sufficient mortar-substrate adhesion when the mortar is applied in thin layers on highly absorbent substrates. Other properties are also affected by $C E$, such as the delay of hydration and setting [2-3]. Some HPMC, HEMC and HEC were characterized in terms of their effect on mortar water retention [4-5]. A few recent works have been concerned with the remarkable mechanism of water retention capacity. A significant influence of the polymer molecular parameters like molecular weight and substitution degrees was evidenced [5], but no clear water retention mechanism could be clearly identified. During evaporation, no admixture loss is occurring, due to the very low vapour pressure of this admixture and a decrease in the water chemical potential occurs [6]. A decrease of water mobility due to the jamming of the diffusion space by the polymer molecules is even expected [7, 8]. However, a recent pulsed gradient field NMR study proved that the macroscopic bulk water self diffusion coefficient is not modified in $C E$ solution or in admixed cement pastes [9]. Moreover, the interdiffusion imaging experiments demonstrated also that the water diffusion at the paste interface is not affected by the presence of cellulosic admixture [9]. So, the possibility of a potential microscopic diffusion barrier specifically at the cement hydrates interfaces is still 
controversial and the origin of the mechanism of water retention due to $C E$ admixture is still an open question.

The aim of the present work is precisely to answer the latter question and to propose a realistic mechanism for the water retention in presence of $C E$ in mortars. To limit the interaction between mineral components, the system was reduced to a white cement paste. We propose another NMR measurement to sense the proton species dynamics specifically at the solid interface. Our primary measurement here is the magnetic field dependence or relaxation dispersion of the proton spin-lattice relaxation rate constant $1 / T_{1}$ (NMRD, $\left.[10,11]\right)$ in neat (without $C E$ ) and $C E$-admixed white cement pastes. For cement pastes, the NMRD technique is neither invasive nor destructive because one measures only the proton NMR response coming from the mixing water itself. This technique has proven useful to give a direct reliable value of the specific surface area of a cement-based material [12]. The remarkable features of the relaxation dispersion support an interpretation in terms of coupled solid/liquid relaxation at pore interfaces, surface diffusion and nuclear paramagnetic relaxation. The measurement is sufficiently fast to be applied continuously during the progressive hydration and setting of the material. In this study, we show the time evolution of the NMR-based specific surface area and the amount of water transiently present at the solid and growing interface. We also discuss the effects of the different $C E$ and Starch Ether $(S E)$ used on the surface diffusion coefficient and on the relative amount of water transiently present at the solid and growing interface.

\section{Experiments}

\subsection{Mineral and organic products}

Cement pastes were prepared with white cement in order to facilitate NMR relaxation experiments. However, we showed before by electronic spin resonance (ESR) that 
paramagnetic $\mathrm{Fe}^{3+}$ ions were clearly present in white cement (see Fig. 6 in ref. [12]). The amount of such paramagnetic ferric ions was quantitatively evaluated through a double integration of this calibrated ESR spectrum [12]. Chemical analysis of this cement was performed by X-ray fluorescence spectroscopy (XRF). The phase compositions were calculated using Bogue's formula with a correction on $\mathrm{CaO}$ due to sulfates [13]. The cement composition is given in Table 1.

The admixtures used are cellulose ethers $(C E)$ of chemical structures given in Fig. 1. The admixture amount was equal to $0.27 \%$ (compared to cement). The characteristics of the main physical-chemistry properties and labels used for the cellulose ethers studied are summarized in Table 2. The number of substituted hydroxyl groups per anhydroglucose unit is expressed as degree of substitution $(D S)$. Moreover the molar ratio of alkoxy groups in the side chains to cellulose is expressed as the average molecular substitution $(M S)$ [14]. The cellulose ether weight-average molecular mass $\left(\bar{M}_{w}\right)$ was determined using size exclusion chromatography $[5,15]$. We have prepared different samples with increased molecular mass $\bar{M}_{w}=$ $\{225,630,910\} k D a$ labeled J1, J2 and J3, respectively (Table 2). Others starch ethers were also investigated, those are polymers providing very different water retentions (Table 3 ).

\subsection{Water retention}

For water retention measurements, mortars were prepared according to the CEReM mixture proportions consisting in $65 \%$ sand, $30 \%$ ordinary Portland cement (CEM I 52.5 R), $5 \%$ calcareous filler with a water to cement ratio $w / c=1$ [16]. Mixing procedure was in accordance with EN 196-1 [17]. Admixture amount (0.27\%) was in addition to the total dry mixture (i.e. cement, sand and filler). The water retention capacity was assessed using the test from an American standard (ASTM C1506-09), based on application of an air depression [18]. It is also used in France for assessing one-coat renderings [19]. 
116 of a perforated dish attached to a vacuum assembly by a funnel. The dish was filled in with

117 the freshly mixed mortar and the vacuum was adjusted to maintain a depression of $50 \mathrm{~mm} \mathrm{Hg}$

118 for $15 \mathrm{~min}$. The initial weight of mixing water is labeled as $W_{0}$. Its loss $\left(W_{1}\right)$ is weighted after

119 the depression period. Hence, the water retention $W R$ was defined by the following equation:

$$
\mathrm{WR}(\%)=\frac{\mathrm{W}_{0}-\mathrm{W}_{1}}{\mathrm{~W}_{0}} \times 100
$$

\subsection{Experiments of proton nuclear magnetic relaxation dispersion (NMRD) in admixed}

We performed proton nuclear magnetic relaxation dispersion (NMRD) on a fast-field

cycling spectrometer from Stelar s.r.l., Mede, Italy, where the polarization magnetic field is

$0.5 \mathrm{~T}$ while the evolution magnetic field ranges from $0.25 \mathrm{mT}$ to $0.5 \mathrm{~T}$. All samples were introduced into a $7 \mathrm{~mm}$-tube which was inserted into a $10 \mathrm{~mm}$-diameter standard NMR tube. Cement pastes were prepared with a water/cement ratio $w / c=0.4$ in order to have an appropriate consistency and for facilitating the NMR measurements at low fields. The proton nuclear magnetization originally at thermal equilibrium is oriented toward the external steady

131 polarized magnetic field (typically $0.5 \mathrm{~T}$ ). The external field is instantaneously decreased (in

132 about $3 \mathrm{~ms}$ ) at an evolution magnetic field chosen in the range from $0.25 \mathrm{mT}$ to $0.5 \mathrm{~T}$ in which

133 the magnetization relaxes, then the magnetic field is fixed to $0.25 \mathrm{~T}$ in which a classical free

134 induced longitudinal magnetization decay measurement is then achieved by applying a $\pi / 2$

135 pulse. Such a fast field cycling sequence is very well documented $[10,11]$. The temperature

136 was fixed at $298 \mathrm{~K}$. The experiment was repeated over a large range of proton Larmor

137 frequencies $\omega_{I} / 2 \pi(10 \mathrm{kHz}-20 \mathrm{MHz})$ in order to obtain the complete dispersion profile of the

138 longitudinal spin-lattice relaxation rate $R_{l}\left(\omega_{I}\right)=1 / T_{1}\left(\omega_{I}\right)$. 
Basically, proton NMR relaxation is a stimulated (non spontaneous) phenomenon driven

140 by the coupling of the proton spins to the magnetic noise induced by molecular motions

141 (translation, rotation, exchange, etc). Varying the magnetic field changes the proton Larmor

142 frequency $\omega_{I} / 2 \pi$, and thus allows exploring the time scales of the magnetic fluctuations

143 (noise) to which the nuclear spin relaxation is sensitive. For diffusive liquids, NMRD gives

144 also a rich source of dynamical information over a large range of length scales, from localized

145 and fast motions at large frequency to a delocalized and slow motions at low frequency.

146 We propose two kinds of NMRD experiments for white admixed cement pastes. The first

147 experiment consists of measuring the magnetic field dependence of the proton spin-lattice

148 relaxation rate $R_{l}\left(\omega_{I}\right)=1 / T_{1}\left(\omega_{I}\right)$ of various admixtures of different $C E$ (Figs. 3, 4). The

149 second experiment consists in probing continuously the spin-lattice relaxation rate $1 / T_{1}$

$150\left(\omega_{I}=2 \pi \times 10 \mathrm{kHz}, t_{\text {hydr }}\right)$ at a fixed and low frequency $(10 \mathrm{kHz})$ during the inducing period of

151 cement paste with various admixtures of different $C E$ (Figs. 5, 7-9). During this period, we

152 observe a monoexponential longitudinal magnetization decay that rules out any distribution of

$153 T_{1}$, the non exponential relaxation only appearing after ten hours of hydration [see Fig. 7 of

154 12]. The continuous measurement of $1 / T_{1}\left(\omega_{I}=2 \pi \times 10 \mathrm{kHz}, t_{\text {hydr }}\right)$ in presence of various

155 admixtures of cellulose ethers allows us to monitor the evolution of the specific surface area

$156 S\left(t_{h y d r}\right) / V$, of the material during the hydration.

\section{3. Theory of nuclear magnetic relaxation dispersion (NMRD) in cement pastes}

158 We proposed previously a theoretical model necessary for interpreting all the NMRD 159 relaxation features reported in Figs. 3 and 4 [12, 20-22]. In the following, we just present a

160 self-contained outline of the basic hypothesis and equations of the model needed for probing

161 the specific surface area and the water surface diffusion coefficient at the growing interface of 162 the adjuvanted cement paste. 
163 (i) Basically, when considering the nuclear relaxation of water embedded in solid hydrated 164 cement, there are coupled relaxation equations for the solid and liquid magnetizations at pore interfaces. The return to equilibrium of either solid or liquid proton spin magnetization is thus a bilinear combination of exponentials with the rate constants for slow $\left(R_{\text {slow }}\right)$ and fast $\left(R_{\text {fast }}\right)$ components $[23,24]$ given by:

$$
\underset{\text { fast }}{R_{\text {flow }}}=\frac{1}{2}\left\{R_{1, s}+R_{1, w}+k(1+1 / F) \mp\left[\left[R_{1, s}-R_{1, w}-k(1-1 / F)\right]^{2}+4 k^{2} / F\right]^{1 / 2}\right\}
$$

Here $R_{1, s}=1 / T_{1, s}$ and $R_{1, w}\left(\omega_{I}\right)$ are the spin-lattice relaxation rate constants associated to

(ii) In most applications of field cycling experiments, the rapidly decaying component $R_{\text {fast }}$ of

(iii) The molecular exchange between the solid and liquid phases is sufficiently fast compared to their respective individual proton relaxation times that a single $R_{l, w}\left(\omega_{I}\right)$ exists given by a linear combination of a bulk and a surface contributions [25]. The latter is weighted by the surface to volume ratio $S / V$ present at a given time of hydration at the solid/liquid interface [20].

(iv) The spin-lattice relaxation rate $R_{1, w}\left(\omega_{I}\right)$ for the confined proton-liquid has a bilogarithmic frequency dependence [26] that comes unambiguously from the two dimensional diffusion of the water molecules along the pore surface modulating the dipole-dipole interaction between the proton species and the paramagnetic $F e^{3+}$ ions fixed at the surface 
$[12,20-22]$. On the contrary, in the frequency range studied, the spin-lattice relaxation rate for the solid protons $R_{1, s}$ does not depend on the frequency [20] and the bulk relaxation rate $R_{1, \text { bulk }}$ is frequency independent [27].

(v) At high frequencies $(10-100 \mathrm{MHz})$, the nuclear paramagnetic relaxation controls the proton relaxation [28]. This gives a typical bump in the NMRD profiles in this frequency range [29] that is not seen here.

(vi) The conservation of these frequency dependencies during the progressive hydration and the evolution of $1 / T_{1}\left(\omega_{I}, t_{\text {hydr }}\right)$ with the hydration time allowed us to renormalize the NMRD data to a single master curve $1 / T_{1}\left(\omega_{I}, t_{\text {hydr }}\right) \propto\left[S_{P, N M R}\left(t_{\text {hydr }}\right)\right] f\left(\omega_{I}\right)$ [20], where $S_{p, N M R}$ is a NMR-based specific surface area of the hydrated cement that appears to be directly proportional to the degree of advancement of chemical reaction [20]. expression of $R_{1, w}$ allows us to reproduce all the observed frequency features:

$$
\begin{aligned}
R_{1, w}\left(\omega_{I}>\omega_{d}\right)= & R_{1 b u l k}+\frac{x \varepsilon}{60} \sigma_{S} \rho_{w} S_{p, N M R}\left(\gamma_{I} \gamma_{S} \hbar\right)^{2} S(S+1) \times \\
& \left\{\begin{array}{l}
\frac{\pi}{\varepsilon^{4}} \tau_{m}\left[3 \ln \left(\frac{1+\omega_{I}^{2} \tau_{m}^{2}}{\left(\tau_{m} / \tau_{s}\right)^{2}+\omega_{I}^{2} \tau_{m}^{2}}\right)+7 \ln \left(\frac{1+\omega_{S}^{2} \tau_{m}^{2}}{\left(\tau_{m} / \tau_{S}\right)^{2}+\omega_{S}^{2} \tau_{m}^{2}}\right)\right] \\
+\frac{8 n \varepsilon^{2}}{r_{I S}^{6}} \tau_{c}\left[\frac{7}{1+\omega_{S}^{2} \tau_{c}^{2}}+\frac{3}{1+\omega_{I}^{2} \tau_{c}^{2}}\right]
\end{array}\right\}
\end{aligned}
$$

203 where $\rho_{w}$ is the density of the water, $\mathcal{E}=3.0 \AA$ is the water molecule size, $x \mathcal{E} \sim 10 \AA$ is an 204 interfacial water layer according to previous calorimetry studies [30] and $r_{I S}=2.7 \AA$ is the 205 distance of minimal approach between $I$ and $S$ spins. The electronic spin $S=5 / 2$ for $F e^{3+} ; n \approx$ 2061 is the number of bounded water molecules in the ligand field of the $\mathrm{Fe}^{3+}$ ions. Since the gyromagnetic ratio of the electron, $\gamma_{S}$, is much larger than that of the proton, $\gamma_{I}\left(\gamma_{S}=658.21\right.$ 
$\left.\gamma_{\mathrm{I}}\right)$, the electronic frequency is $\omega_{S}=658.21 \omega_{\mathrm{I}}$. In Eq. $3, N_{S} / N=x \varepsilon \rho_{w} S_{p, N M R}$ represents the ratio

209 of the number of water molecules transiently present at the pore surface, $N_{S}$, to the total 210 number, $N$, of exchangeable water molecules in the sample. Also in Eq. (3), $\tau_{m}$ is the 211 correlation time characterizing the two-dimensional diffusion of the proton species at the 212 surface of the pores. We introduce also the effects of the finite time of residence $\tau_{S}>>\tau_{m}$ for 213 the mobile proton species at the surface of the pores by an exponential cut-off in the time 214 dependence of the pair correlations IS. The correlation time $\tau_{c}$ of the nuclear paramagnetic 215 relaxation is given by $1 / \tau_{c}=1 / \tau_{e x}+1 / T_{1 F e} \approx 1 / T_{1 F e}$ where $\tau_{e x}\left(\tau_{e x}>>T_{1 F e}\right)$ is the lifetime of water 216 in the ligand field of the ferric ions. The electronic spin-lattice relaxation time of the 217 paramagnetic impurity $T_{1 F e}$ (of the order of $210^{-11} \mathrm{~s}$ ) is constant at low frequency and has a 218 frequency dependence at higher frequency defined in ref [12, 20, 28]. The relaxation rate in 219 the bulk phase, $R_{1 b u l k} \sim 0.3 s^{-1}$ is caused by the fast molecular reorientations and translations 220 and is independent of frequency in the low field range studied [27]. Last, Substituting Eq. (3) 221 into Eq. (2) gives the theoretical expression that we can compare with the experiments in Figs. $222 \quad 3$ and 4.

\section{Results and discussion}

\subsection{Water retention of freshly-mixed mortars}

The water retention capacity of each admixed mortar was evaluated; the results are presented in Table 2 and 3. The cellulose ethers improved water retention up to $98.9 \%$ for HPMC J3. With respect to the water retention capacity of the non-admixed mortar $(64.5 \%)$, this represents a very large increase. However the starch ethers procure very different water retention capacities ranging from $66.2 \%$ to $92.6 \%$. 


\subsection{Surface diffusion coefficient}

231 The surface diffusion coefficient is obtained from a fitting procedure with Eqs. (2) and (3) 232 on the NMRD profiles of Figs. 3 and 4. Fig. 3a shows the NMRD profiles for the neat 233 (without $C E$ ) white cement paste. Fig. 3b shows the NMRD profiles of a white cement paste 234 admixed with HPMC J3. On both cases, we have varied the duration of the experiments by 235 changing the number of frequencies explored. Using thirty points takes some time (about 5 236 minutes per point) and we note that frequency profiles were affected by the kinetics of cement 237 hydration. Indeed, the time needed to register one complete profile is about $2 \mathrm{~h} 30$. In consequence, we could not observe the characteristic 10/3 slope ratio of the bilogarithmic frequency dependence usually found in cement based materials [20]. A simple procedure that 240 allows limiting such a kinetic effect during the measurements is to decrease the number of 241 frequency points on the profiles. One observes on Figs. 3a, b that reducing the experimental time to 45 minutes allows decreasing the kinetic effect. For instance, we observe that the first

24312 points almost merge at high frequency, then, the gap between the two profiles increases 244 with time. In consequence, all NMRD dispersion curves of this work were obtained with 245 twelve different and logarithmically spaced frequency values between 0.01 and $15 \mathrm{MHz}$ to 246 avoid the problems due to kinetics of cement hydration. This procedure allows a quite 247 reasonable fit of the plateau dependence at low frequency and the bilogarithmic dependence at 248 higher frequency (Figs. 3 and 4).

249 Fig. 4a presents the proton NMRD data obtained for different admixed cement pastes with 250 three HPMC (J1, J2 and J3). The correlation time $\tau_{m}$ is determined using the model described 251 above. For these four different cement pastes, we find similar results for the translational 252 surface diffusion approximately equal to $\tau_{m} \approx 1 n s$ and $\tau_{s} \approx 10 \mu$ s This suggests a pore scale 253 invariance of water dynamics at the pore surface in C-S-H whatever the paste composition. 254 This value of $\tau_{m}$ is in agreement with previous studies performed on cement pastes or on 
mortars $[20,21]$. The translational diffusion coefficient $D_{\text {surf }}$ at the pore surface can be 256 deduced from the Stokes-Einstein relationship: $D_{\text {surf }}=\varepsilon^{2} /\left(4 \tau_{m}\right)$ where $\varepsilon=3 \AA$ is the water 257 molecules size. For all cement pastes (admixed or not), the surface diffusion coefficient is 258 about $D=2.2510^{-11} \mathrm{~m}^{2} . \mathrm{s}^{-1}$ i.e. about $1 / 100$ of that of bulk water at $23^{\circ} \mathrm{C}$. In consequence, 259 the presence of cellulose ethers in cement paste does not modify the surface diffusion 260 coefficient at the pore surface. This result is similar to those obtained in the macroscopic scale 261 by pulsed field gradient NMR showing that the CE does not modify the diffusion coefficient 262 of water in the bulk [9].

263 The effect of starch ethers was also investigated with M1 and M4. These polymers provide 264 very different water retentions ( $92.6 \%$ and $66.2 \%$ respectively). The protocol was identical to 265 that of cement pastes containing cellulose ethers. The results are shown on Fig. 4b. The 266 translational correlation times are also $\tau_{m} \approx 1 n s$ and $\tau_{s} \approx 10 \mu$ for the admixed cement paste 267 with M1 and M4 respectively. This demonstrates that the translational diffusion coefficient at 268 the surface of the solid interfaces is not affected by starch ether's presence.

269 These experiments thus demonstrate that cellulose and starch derivatives do not change the 270 surface diffusion coefficient of water. The water mobility at pore surface is thus not modified 271 in presence of such polymers.

\subsection{Specific surface area and relative quantity of water transiently present at pore surface}

On Figs. 3 and 4, one observes a constant value for $1 / T_{1}$ when the proton frequency

274 becomes $\omega_{I<} \omega_{d}=2 \pi \times 30 \mathrm{kHz}$. Such a cross-over frequency $\omega_{d}$ is of the order of the dipolar 275 interaction in the solid state between two protons separated by $0.155 \mathrm{~nm}$. On this low 276 frequency range, the confined proton liquid explores relaxation processes induced by 277 characterized long correlation times encountered in the solid state: $\tau_{\text {rigid }}=1 / \omega_{d}=5.3 \mu s$. We 278 have shown in Section 3 (i) that owing to the cross relaxation process, the intermolecular 279 dipole-dipole interaction then couples the water spin relaxation to that of the solid and the 


$$
R_{\text {slow }}\left(\omega_{I}=2 \pi \times 10 k H z, t_{h y d r}\right) \approx R_{1, s}\left(t_{h y d r}\right) \approx R_{1, w}\left(\omega_{I}=\omega_{d}, t_{h y d r}\right)
$$

$$
R_{\text {slow }}\left(\omega_{I}<\omega_{d}\right) \approx R_{1, s}+k / F \text {, with } R_{1, s} \equiv R_{1, w}\left(\omega_{I}=\omega_{d}\right)=\text { Cte }
$$

magnetic field dependence of the immobilized spin system. From a mathematical point of view, Eq. (2) tends to the constant relaxation rate of the solid matrix $R_{l s}$ when the dipolar cross-relaxation rate is lower than the individual relaxation rates, i.e.: $k \ll R_{1, w}, R_{1, s}$ and $\left|R_{1, w}-R_{1, s}\right|$ and providing that $F<<1$ and $k / F>>k$ :

The observed data presented in Fig. 5 confirm the plateau observed at low frequency (at 10 $\mathrm{kHz}$ ) for $R_{\text {slow }}$ during the first hundred minutes. Then, relaxation rate increases due to cement hydration and development of the specific surface area. Moreover, a zoom on the Fig. 5 shows that small but significative differences exist between each adjuvanted cement pastes. The observed plateau for frequency below $\omega_{d}$ is thus characteristic of the rigid-lattice limit of the solid-proton hydrates. The absolute value of such a plateau, measured for instance at 10 $\mathrm{kHz}$, is thus indicative of the specific surface area precisely at the solid-liquid interface:

As the correlation times $\tau_{m}$ and $\tau_{s}$ are constants whatever the cement paste composition, the continuous measurement of $R_{\text {slow }}\left(t_{\text {hydr }}\right)$ at a fixed and low frequency $\omega_{I}=2 \pi \times 10 \mathrm{kHz}$, allows to probe directly the time evolution of the specific surface area of the cement paste $S_{p, N M R}$ :

$$
S_{P, N M R}\left(t_{h y d r}\right)=\frac{1 / T_{1, \text { observed }}\left(10 \mathrm{kHz}, \mathrm{t}_{\text {hydr }}\right)}{\frac{x \varepsilon \pi}{60 \varepsilon^{4}} \sigma_{S} \rho_{w}\left(\gamma_{I} \gamma_{S} \hbar\right)^{2} S(S+1) \tau_{m}\left[3 \ln \left(\frac{1+\omega_{d}^{2} \tau_{m}^{2}}{\left(\tau_{m} / \tau_{s}\right)^{2}+\omega_{d}^{2} \tau_{m}^{2}}\right)+7 \ln \left(\frac{1+659^{2} \omega_{d}^{2} \tau_{m}^{2}}{\left(\tau_{m} / \tau_{s}\right)^{2}+659^{2} \omega_{S}^{2} \tau_{m}^{2}}\right)\right]}
$$


The continuous measurement of $1 / T_{1}\left(\omega_{I}=2 \pi \times 10 \mathrm{kHz}, t_{\text {hydr }}\right)$ in presence of various

admixtures of cellulose ethers thus allows to monitor the evolution of the specific surface area $S_{P, N M R}\left(t_{\text {hydr }}\right)$, of the material during the hydration.

\subsection{How NMR relaxation can elucidate water retention mechanisms}

In the very early aged of the hydration, the amount of CSH is not very large and the admixed polymer is still in the interstitial solution within the pores of the paste, even if the cellulose ethers are rather large. The relaxation of the mobile proton-water thus will be influenced by the presence of the polymer. We show that measuring the time evolution of the spin-lattice relaxation rate $R_{\text {slow }}\left(10 \mathrm{kHz}, t_{\text {hydr }}\right)$ at $10 \mathrm{kHz}$, especially in the first minutes of hydration allows probing quantitatively the relative population of water transiently present at the solid hydrate surfaces $N_{S} / N\left(t_{\text {hydr }}\right)$.

On Fig. 5, the continuous lines exhibit the time evolution of $R_{\text {slow }}\left(10 \mathrm{kHz}, t_{\text {hydr }}\right)$ for the non adjuvanted (neat) white cement and with HPMC J1, J2, J3 admixtures. The periods $d_{C E}$ during which the plateau remains constant are evidenced ( $d_{0}$ is related to neat cement) and ordered as followed:

$$
d_{0}=11 \mathrm{~min}<d_{J 3}=60 \mathrm{~min}<d_{J 2}=79 \mathrm{~min}<d_{J 1}=107 \mathrm{~min} .
$$

This period $d_{C E}$, measured at low frequency, is a clear indicator of the delay of hydration induced by the $C E$ polymer introduced into the material. The fact that $d_{0}$ stays much smaller than the other $d_{C E}$ is a clear indication of the delayed hydration and setting induced by the HPMC J1, J2, J3 admixtures. We show on Fig. 6, that one can correlate these delays of hydration measured by NMRD to the ones measured from isotherm calorimetry measurements (determined using the method described in ref. [31]) on similar materials. The quasi linear dependence $\left(r^{2}=0.99\right)$ shows very good accuracy between the microscopic (NMRD) and macroscopic (calorimetry) measurements. 
Another important parameter can be extracted from the behaviour of the time evolution of $R_{\text {slow }}\left(10 \mathrm{kHz}, t_{\text {hydr }}\right)$ during the first 100 minutes of hydration. This is the value of the plateau

itself, noted, $R_{C E}=R_{\text {slow, observed }}\left(10 \mathrm{kHz}, t_{\text {hydr }}\right)$ which depends critically on the nature of the admixtures. The results found for the neat white cement paste and HPMC-admixed cement pastes $\mathrm{J} 1, \mathrm{~J} 2, \mathrm{~J} 3$ are ordered as followed:

$$
R_{0}=30 s^{-1}<R_{J 1}=35,9 s^{-1}<R_{J 2}=36,3 s^{-1}<R_{J 3}=37,6 s^{-1}
$$

where $R_{0}$ is the value of the low-frequency plateau for neat cement. According to Eqs. (2) and (3), $R_{C E}$ is proportional to the correlation time $\tau_{m}$ and directly related to the relative amount of water $N_{S} / N$ transiently present during the first 100 minutes at proximity of solid interfaces that is proportional to the specific surface area:

$\frac{N_{S}\left(t_{h y d r}<d_{C E}\right)}{N}=x \varepsilon \rho_{w} S_{p, N M R}\left(t_{h y d r}<d_{C E}\right)$

where $S_{P, N M R}\left(t_{h y d r}\right)$ is defined in Eq. (6). Substitution of all the parameters measured or defined above in Eq. (7) and owing to the observation that $\tau_{m} \approx 1 n s$ and $\tau_{s} \approx 10 \mu$ se universal for all the cement-based materials, one obtains a proportion $N_{S}\left(t_{h y d r}=2 \mathrm{~min}\right) / \mathrm{N}=1.06 \%$ for the neat white cement paste and $N_{S}\left(t_{h y d r}=2 \mathrm{~min}\right) / N=1.36 \%$ for the admixed cement pastes with HPMC J3. One obtains intermediate values for the other admixtures HPMC J1 and HPMC J2. The fact to probe, in the first minutes of cement hydration, the fraction of mobile water molecules transiently present at solid interfaces $N_{S} / N$ in presence of different nature and quantity of cellulose ethers is a key result of this study that can be directly linked to the relative amount of water retained on the pores surface.

We have applied the same NMRD method for starch ethers M1, M4 (Fig. 7) and found the 349 following results: $d_{0}=11 \mathrm{~min}<d_{M 4}=19 \mathrm{~min}<d_{M 1}=22 \mathrm{~min}$ and $R_{0}=30 \mathrm{~s}^{-1}<$ 
$R_{M 4}=33.6 \mathrm{~s}^{-1}<R_{M 1}=35.2 \mathrm{~s}^{-1}$ that reveal a much weaker microscopic water retention effect than the ones obtained with $C E$ polymers.

Moreover, the effect of the substitution of $C E$ on the water transiently present at the solid interfaces for HEMC C4 and HEC N7 molecules is shown on Fig. 8. These two polymers have been chosen to evidence the effect of hydrophobic methoxyl groups present on HEMC and absent on HEC. We check that the ASTM method cannot discriminate the important water retention (WR=98.8\%) for the cements adjuvanted by these two polymers [31]. We remind that this discrimination is limited by the depression imposed by the method (50 mmHg). To improve the sensibility of the ASTM method, one should increase largely such a depression. On the contrary, we show here that the NMRD data can indeed discriminate the 360 observed value $R_{C E}=39 s^{-1}$ for the hydrophilic HEC N7 molecule and $R_{C E}=37 s^{-1}$ for the 361 hydrophobic HEMC molecule, because NMR is very sensitive to the local environment of the 362 proton spins. So the macroscopic ASTM measurement is not able to distinguish polymer's effect while NMRD is more sensitive to the local physical-chemistry at the solid interfaces.

364 Last, Fig. 9 shows the NMRD results for HPMC J1 with two different concentrations namely 0.1 and $0.4 \%$ giving 82.3 and $98.0 \%$ macroscopic water retention, respectively. As 366 expected, the value of the plateau $R_{C E}$ increases significantly with the concentration of 367 admixture. Finally, on Fig. 10, the linear correlation $\left(\mathrm{r}^{2}=0.93\right)$ of $R_{C E}$ and water retention of 368 mortar admixed with three different concentrations $0.1,0.27$ and $0.4 \%$ of HPMC J1 proves 369 that our microscopic NMRD measurements on cement pastes could be correlated with the 370 macroscopic water retention on mortars.

\section{5. Conclusion}

372 An original method based on two different applications of proton-water magnetic 373 relaxation dispersion (NMRD) has been proposed to elucidate the effect of cellulose ethers $374(C E)$ on water retention of freshly-mixed white cement paste. 
The first application of NMRD probes directly the proton-water surface dynamics from the magnetic field dependence of the nuclear spin-lattice relaxation rate. In spite of modifications of the solution's viscosity, we find that the cellulosic derivatives do not modify the surface diffusion coefficient of water that is about two orders of magnitude smaller than that of bulk water.

380 The second application of NMRD concerns the continuous measurements of the spin381 lattice relaxation rates at a fixed and very low Larmor frequency. At the very early ages, this application probes continuously and for the first time, the relative population of water transiently present at the surface of the time-growing solid hydrate interfaces. Compared to neat white cement, the $C E$ modify significantly the surface fraction of mobile water molecules transiently present initially at solid surfaces. Our results show that the larger the fraction, the

386 better water-retention capacity of the hydrated cement or mortar samples at the proximity of a solid support. This quantity measured for all admixed cement pastes is thus relevant to explain the water retention mechanism. Furthermore, a linear correlation is found between the NMR surface fraction and the standard ASTM method used by the mortar industry to estimate

390 the water retention capacity of admixed mortars. However for very strong water retention, it 391 seems that the ASTM is not able to distinguish the polymer's effect while NMRD enables us 392 to make the difference between two admixtures.

\section{$394 \quad$ Acknowledgements}

395 The authors acknowledge all the industrial and academic CEReM members 396 (http://cerem.cstb.fr). 
399 [1] S. Mansoutre, P. Colombet, H. Van Damme. Water retention and granular rheological behavior of fresh C3S paste as a function of concentration. Cem. Concr. Res., 29 (1999) 1441-1453.

402

403

404

405

406

407

408

409

410

411

412

413

414

415

416

[2] A. Peschard, A. Govin, P. Grosseau, B. Guilhot, R. Guyonnet. Effect of polysaccharides on the hydration of cement paste at early ages. Cem. Concr. Res., 34 (2004) 2153-2158.

[3] J. Pourchez, A. Peschard, P. Grosseau, R. Guyonnet, B. Guilhot, F. Vallée. HPMC and HEMC influence on cement hydration. Cem. Concr. Res., 36 (2006) 1777-1780.

[4] J. Pourchez, B. Ruot, J. Debayle, E. Pourchez, P. Grosseau. Some aspects of cellulose ethers influence on water transport and porous structure of cement-based materials. Cem. Concr. Res., 40 (2010) 242-252.

[5] L. Patural, P. Marchal, A. Govin, P. Grosseau, B. Ruot, O. Devès. Cellulose ethers influence on water retention and consistency in cement-based mortars. Cem. Concr. Res., 41 (2011) 46-55.

[6] H. Kuhn, D. H. Waldeck, H.-D. Försterling. Principles of Physical Chemistry. WileyInterscience (2009).

[7] B. Penke, S. Kinsey, S. J. Gibbs, T. S. Moerland, B. R. Locke. Proton Diffusion and T1 Relaxation in Polyacrylamide Gels: A Unified Approach Using Volume Averaging. J. Magn. Reson., 132 (1998) 240-258.

[8] N. Nestle, A. Kühn, K. Friedemann, C. Horch, F. Stallmach, G. Herth, Water balance and pore structure development in cement materials in internal curing with modified superabsorbent polymer studied by NMR, Microporous and Mesoporous Materials 125 (2009) 51-57.

[9] L. Patural, P. Porion, H.Van Damme, A. Govin, Ph. Grosseau, B. Ruot, O. Devès, A pulsed field gradient and NMR imaging invsertigations of the water retention 
mechanism by cellulose ethers in mortars, Cem. Concr. Res., 40 (2010) 1378-1385.

424

[10] A. G. Redfield, W. Fite, H.E. Bleich, Precision high speed current regulators for occasionally switched inductive loads, Rev. Sci. Instrum., 39 (1968) 710-715.

[11] F. Noack, Basic and novel aspects of NMR field-cycling spectroscopy, Bull. Ampere, 175 (1994) 18-35.

[12] J.-P. Korb, L. Monteilhet, P.J. McDonald, J. Mitchell. Microstructure and texture of hydrated cement-based materials: A proton field cycling relaxometry approach. Cem. Concr. Res., 37 (2007) 295-302

[13] H.F.W. Taylor, Cement Chemistry, $2^{\text {nd }}$ edition, Academic Press Thomas Telford (2007).

[14] F. Crössmann, W. Klaus. Wasserlösliche Celluloseäther im Spiegel der Anwendungstechnik. Collection of publication of a group of authors of Kalle, branch of Hoechst AG, Wiesbaden-Biebrich (1974).

[15] C. Clasen, W.-M. Kulicke. Determination of viscoelastic and rheo-optical material functions of water-soluble cellulose derivatives. Prog. Polym. Sci., 26 (2001) 1839-1919.

[16] B. Ruot, T. Goto, J. Pourchez. Some aspects of cellulose ethers and latexes influence on the properties of cement-based materials - Examples of results obtained within the CEReM. Proceedings of the VII SBTA ( $7^{\circ}$ Symposio Brasileiro De Tecnologia Das Argamassas), (2007).

[17] EN 196-1. Methods of testing cement - Part 1: Determination of strength. (2006).

[18] Standard C1506-09. Standard test Method fo Water Retention of Hydraulic CementBased Mortars and Plasters. Am. Soc. Test. Mat. (2009).

[19] Certifié CSTB Certified des enduits monocouches d'imperméabilisation-Modalités d'essais, DT 9 du 10 avril 2008.

[20] F. Barberon, J.-P. Korb, D. Petit, V. Morin, E. Bermejo. Probing the surface area of a cement-based material by nuclear magnetic relaxation dispersion. Phys. Rev. Lett., 90 
(2003) 116103-116104.

449 [21] J.-P. Korb. NMR and nuclear spin relaxation of cement and concrete materials.

$450 \quad$ Curr. Opin. in Coll. Int. Sci., 14 (2009) 192-202.

451 [22] J.-P. Korb. Microstructure and texture of cementitious porous materials. Magn. Res. Imag., 25 (2007) 466-469.

[23] I. Solomon, Relaxation processes in a system of two spins, Phys. Rev. 99, (1955) 559565.

455

[24] R.G. Bryant, D. A. Mendeslon, C. Coolbaugh Lester, The magnetic field dependence of proton spin relaxation in tissues, Magn. Reson. in Medicine 21, (1991) 117-126.

[25] K. R. Brownstein and C. E. Tarr, Importance of classical diffusion in NMR studies of water in biological cells, Phys. Rev. A, 19, (1979) 2446-2453.

[26] J.-P. Korb, M. Whaley-Hodges, R. G. Bryant. Translational diffusion of liquids at surfaces of microporous materials: Theoretical analysis of field-cycling magnetic relaxation measurements. Phys. Rev. E, 56 (1997) 1934-1945.

[27] A. Abragam, The principles of Nuclear Magnetism, Ch 8, (Clarendon, Oxford, 1961).

[28] N. Bloembergen and L. O. Morgan, Proton relaxation times in paramagnetic solutions; effects of electron spin relaxation, J. Chem. Phys., 34, 842 (1961).

[29] A. Plassais, M.-P. Pomies, N. Lequeux, J.-P. Korb, D. Petit, F. Barberon, 467 Microstructure evolution of hydrated cement pastes, Phys. Rev. E, 72 (2005) 041401041408.

[30] J. J. Fripiat, M. Letellier, and P. Levitz, Interaction of Water with Clay Surfaces, Phil. Trans. R. Soc. A, 311 (1984) 287-299.

[31] L. Patural, PhD Thesis, ENSM (2011). 
471 Table 1. Phase composition of the white cement.

\begin{tabular}{|c|c|c|c|c|c|}
\hline Phases & $\mathrm{C}_{3} \mathrm{~S}$ & $\mathrm{C}_{2} \mathrm{~S}$ & $\mathrm{C}_{3} \mathrm{~A}$ & $\mathrm{C}_{4} \mathrm{AF}$ & Sulfates \\
\hline Phase composition (\%) & $68.8 \pm 0.3$ & $10.4 \pm 0.7$ & $10.5 \pm 0.1$ & $1.0 \pm 0.1$ & $7.5 \pm 0.1$ \\
\hline
\end{tabular}

472 Table 2. Molecular parameters and water retention for cellulose ethers used in this work.

473

474

475

476

477

478

479

480

481

\begin{tabular}{|c|c|c|c|c|c|c|c|}
\hline $\mathbf{C E}$ & $\begin{array}{c}\overline{M_{w}} \\
(\mathbf{k D a})\end{array}$ & $\begin{array}{l}\mathrm{OCH}_{3} \\
(\%)^{\mathrm{a}}\end{array}$ & $D S$ & $\begin{array}{c}\mathrm{OC}_{2} \mathrm{H}_{4} \mathrm{OH} \\
(\%)^{\mathrm{a}} \\
\end{array}$ & $\begin{array}{c}\mathrm{OC}_{3} \mathrm{H}_{6} \mathrm{OH} \\
(\%)^{\mathrm{a}} \\
\end{array}$ & MS & $\begin{array}{c}\text { Water retention } \\
(\%)\end{array}$ \\
\hline HEMC C4 & 380 & 27.4 & 1.7 & 4.80 & - & 0.15 & 98.8 \\
\hline HPMC J1 & 225 & 28.2 & 1.8 & - & 2.98 & 0.10 & 96.8 \\
\hline HPMC J2 & 630 & 28.2 & 1.8 & - & 2.98 & 0.10 & 98.6 \\
\hline HPMC J3 & 910 & 28.2 & 1.8 & - & 2.98 & 0.10 & 98.9 \\
\hline HEC N7 & 2900 & - & - & 56.0 & - & 2.5 & 98.8 \\
\hline
\end{tabular}

${ }^{\mathrm{a}}$ Informations provided by the manufacturer.

$\mathrm{Mw}=$ Cellulose ether weight-average molecular mass determined using size exclusion chromatography DS= Number of substituted hydroxyl groups per anhydroglucose unit expressed as degree of substitution MS=Molar ratio of alkoxy groups in the side chains to cellulose expressed as the average molecular substitution $\mathrm{OCH}_{3}=$ Methoxy group

$\mathrm{OCH}_{2} \mathrm{H}_{4} \mathrm{OH}=$ Hydroxyethyl group

$\mathrm{OCH}_{3} \mathrm{H}_{6}=$ Hydroxypropyl group

Table 3. Molecular parameters and water retention for starch ethers (SE) used in this work.

\begin{tabular}{|c|c|c|c|c|c|}
\hline \multirow{2}{*}{ SE } & \multirow{2}{*}{$\begin{array}{c}\overline{M_{w}} \\
(\mathbf{k D a})\end{array}$} & \multirow{2}{*}{$\begin{array}{l}\text { Amylopectin / } \\
\text { amylose ratio }^{\text {a }}\end{array}$} & \multicolumn{2}{|c|}{ Degrees of polymerization $^{\text {a }}$} & \multirow{2}{*}{$\begin{array}{c}\text { Water retention } \\
(\%)\end{array}$} \\
\hline & & & Amylose & Amylopectin & \\
\hline SE M1 & 860 & $80 / 20$ & 4000 & 2000000 & 92.6 \\
\hline SE M4 & 830 & $80 / 20$ & 4000 & 2000000 & 66.2 \\
\hline
\end{tabular}

${ }^{\mathrm{a}}$ Informations provided by the manufacturer. 
484 Fig. 1. Structure of cellulose ethers (a): HPMC, (b): HEMC, (c): HEC. Substituent positions are arbitrary; they may differ slightly from one molecule to another.

Fig. 2. Experimental device to measure water retention with ASTM C1506-09 standard.

487

488

489

490

491

492

493

494

495

496

497

498

499

500

501 502

503

504

\section{Fig. 3. Measured water ${ }^{1} \mathrm{H}$ magnetic relaxation dispersion profiles for hydrated white cement} paste $(w / c=0.4)$ as a function of the proton Larmor frequency. The experiments have been realized in the early hydration period (0-45 min, filled circles) and on a larger hydration time (up to few hours, filled squares) for neat (without $C E$ ) cement (a) and cement admixed with HPMC J3 (b). The continuous lines are the best fits obtained with Eqs. (2) and (3).

Fig. 4. Measured water ${ }^{1} \mathrm{H}$ spin-lattice relaxation rates of hydrated white cement pastes $(w / c=0.4)$ as a function of the proton Larmor frequency. (a) Effect of three cellulose ethers (HPMC J1, J2, J3). The J3data sets are the same as those in Fig. 3. (b) Effect of two starch ethers (M1 and M4). In both cases, we have reported the results obtained with the neat white cement paste. The continuous lines are the best fits obtained with Eqs. (2) and (3).

Fig. 5. Water ${ }^{1} \mathrm{H}$ spin-lattice relaxation rates of hydrated cement pastes $(w / c=0.4)$ as a function of hydrating time measured at a Larmor frequency of $10 \mathrm{kHz}$. Effect of three cellulose ethers (HPMC, J1, J2, J3). The inset represents a zoom on the frequency range of the plateau.

Fig. 6. Correlation between the delays obtained by the NMRD and isotherm calorimetry.

Fig.7. Water ${ }^{1} \mathrm{H}$ spin-lattice relaxation rates of hydrated cement pastes $(w / c=0.4)$ as a function of hydrating time measured at a Larmor frequency of $10 \mathrm{kHz}$. Effect of three cellulose ethers 
505 (Neat, starch CE: M1, M4). The inset represents a zoom on the frequency range of the , 506 plateau.

507 Fig. 8. Comparison of the water ${ }^{1} \mathrm{H}$ spin-lattice relaxation rates of hydrated cement pastes $508(w / c=0.4)$ as a function of hydrating time measured at a Larmor frequency of $10 \mathrm{kHz}$. Effect 509 of different cellulose ethers (Neat, HEC N7, HEMC C).

510 Fig. 9. Comparison of the water ${ }^{1} \mathrm{H}$ spin-lattice relaxation rates of hydrated cement pastes $511(w / c=0.4)$ as a function of hydrating time measured at a Larmor frequency of $10 \mathrm{kHz}$. Effect 512 of the $C E$ concentration of HPMC.

513 Fig. 10. Correlation between the water retention evidenced from the macroscopic 514 measurement described in Fig. 2 (ASTM C1506-09 standard) and the NMRD method. The 515 different concentrations of HPMC J1 are indicated.

516 
(a)

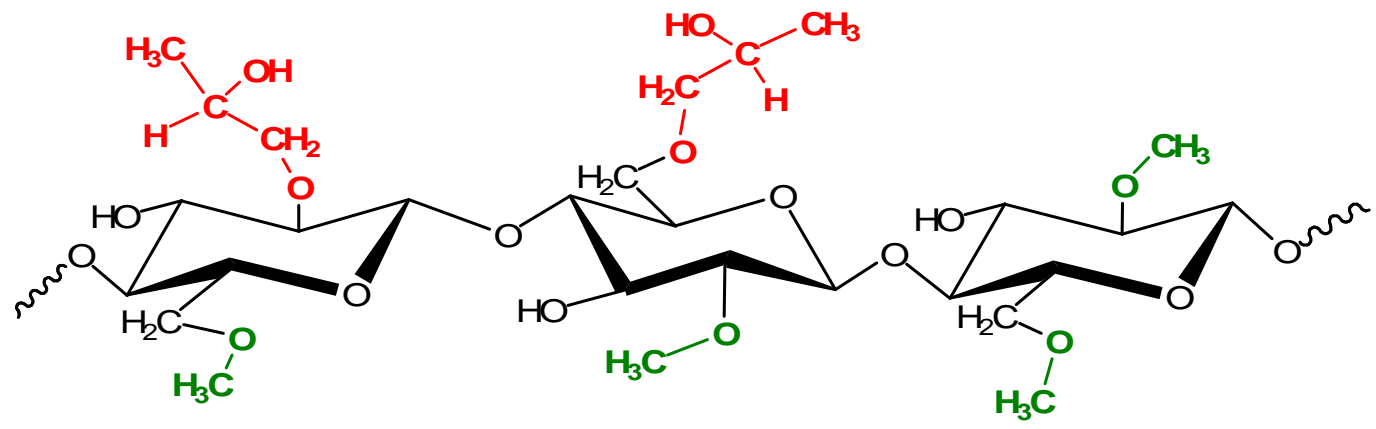

519
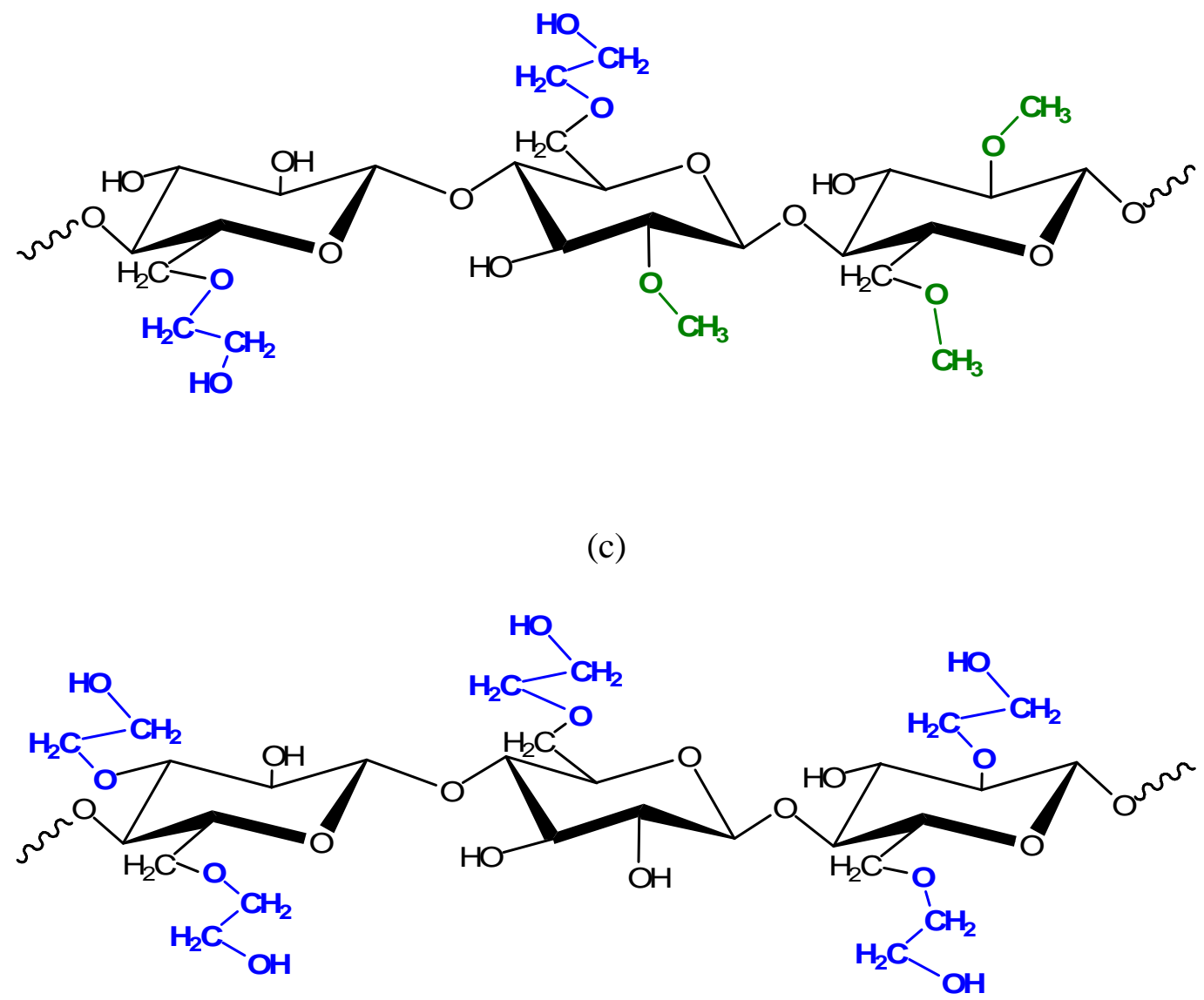

Fig. 1 


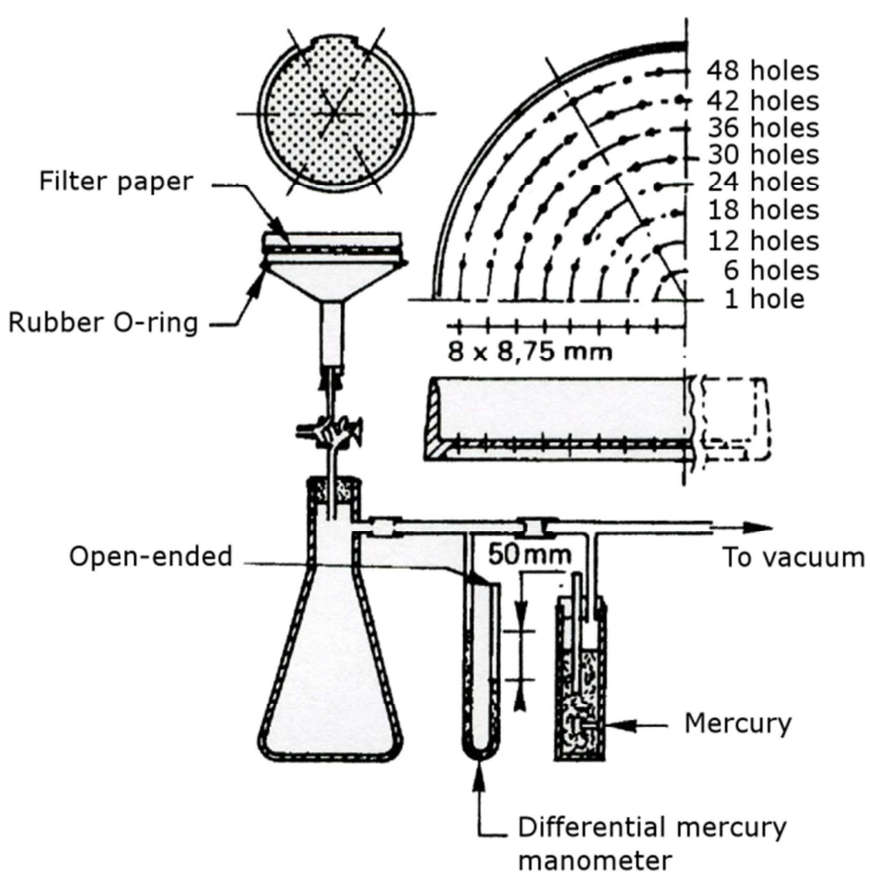

Fig. 2 

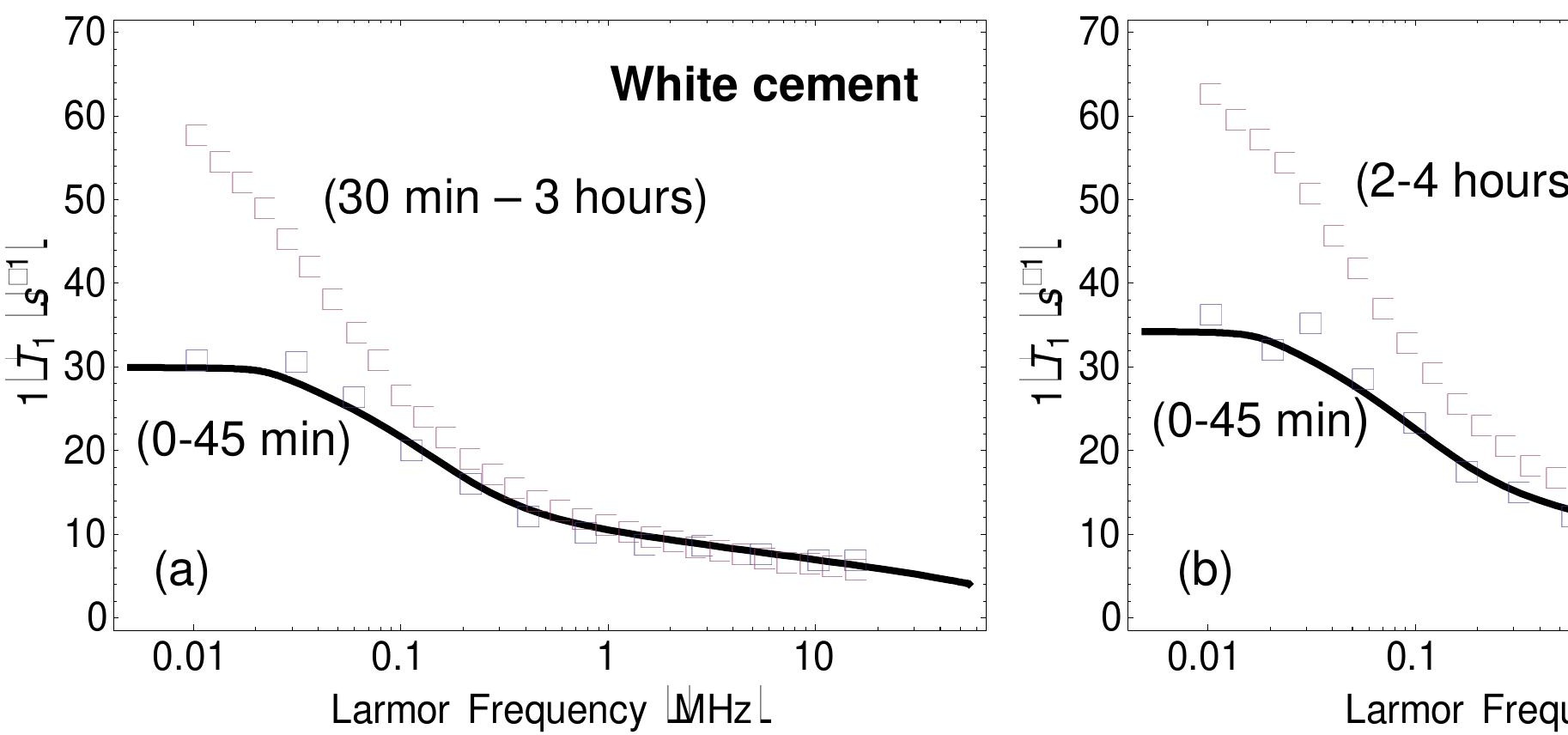
Fig. 3 

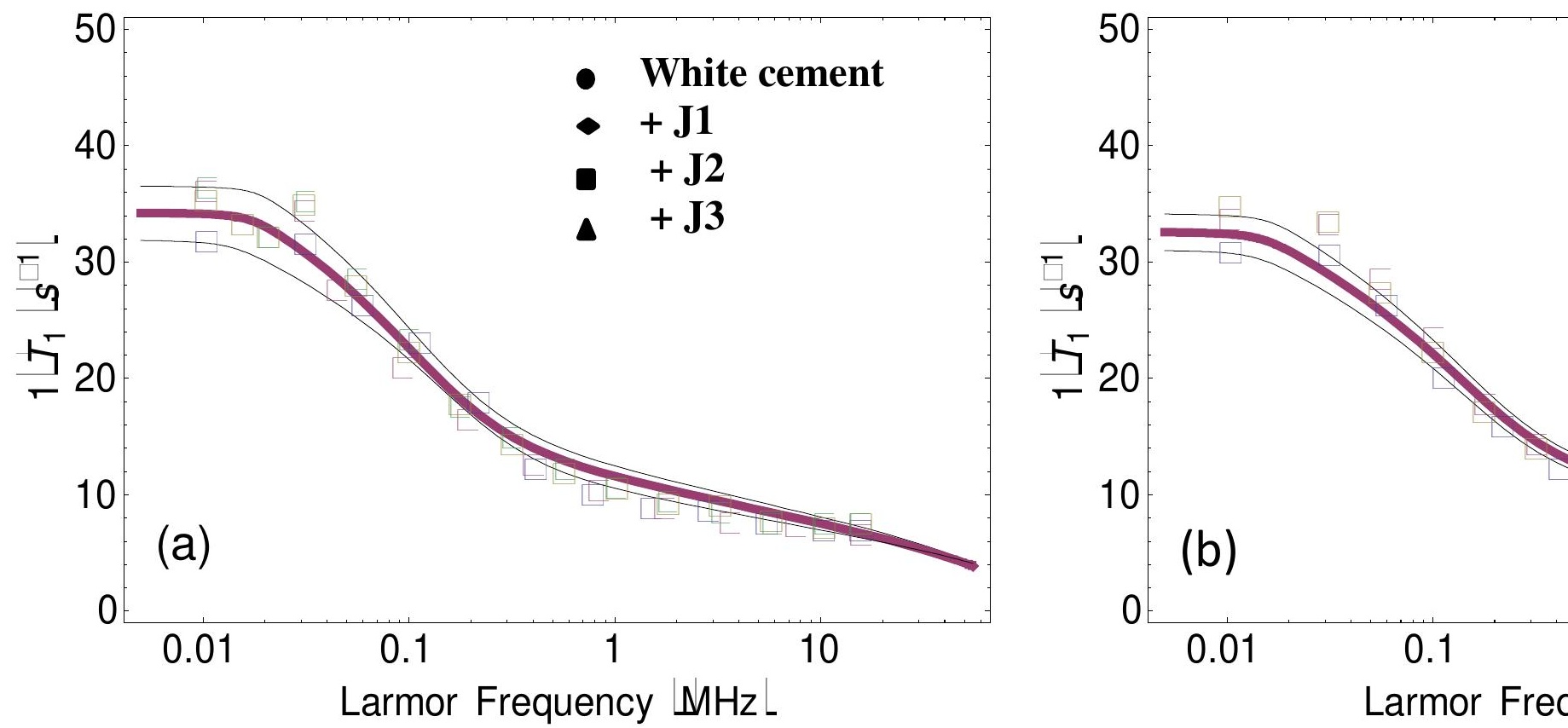

Fig.4

538

539

540 


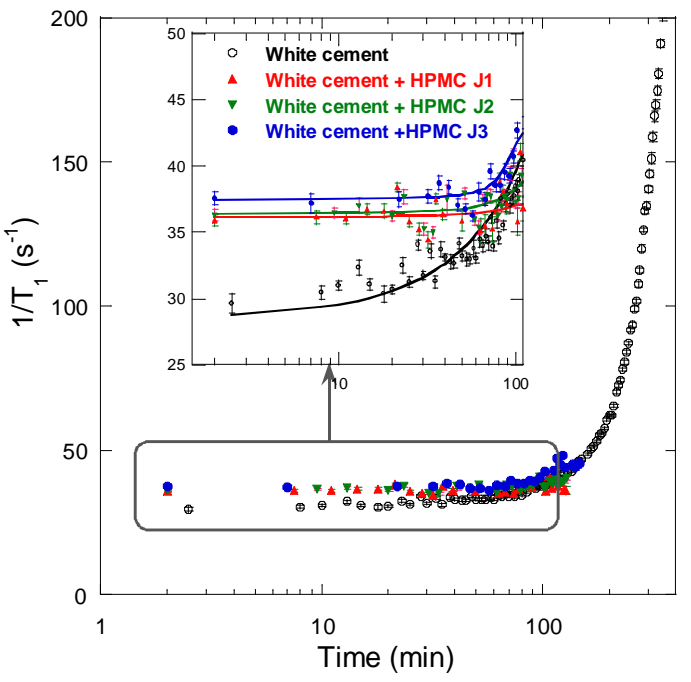

543

Fig. 5 


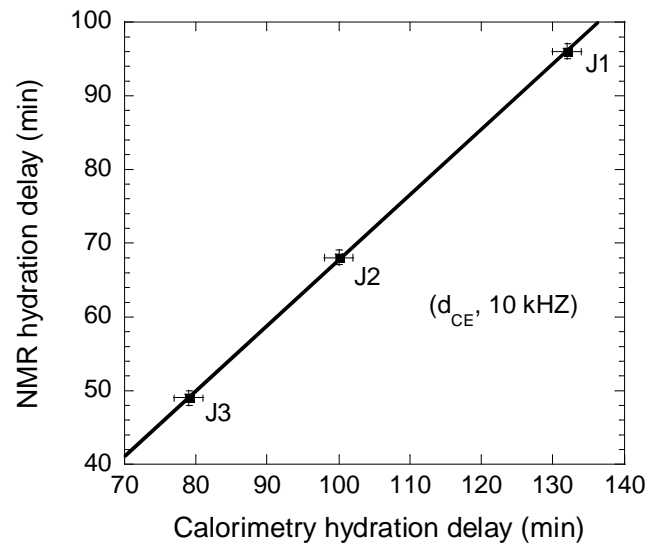

Fig. 6

546 


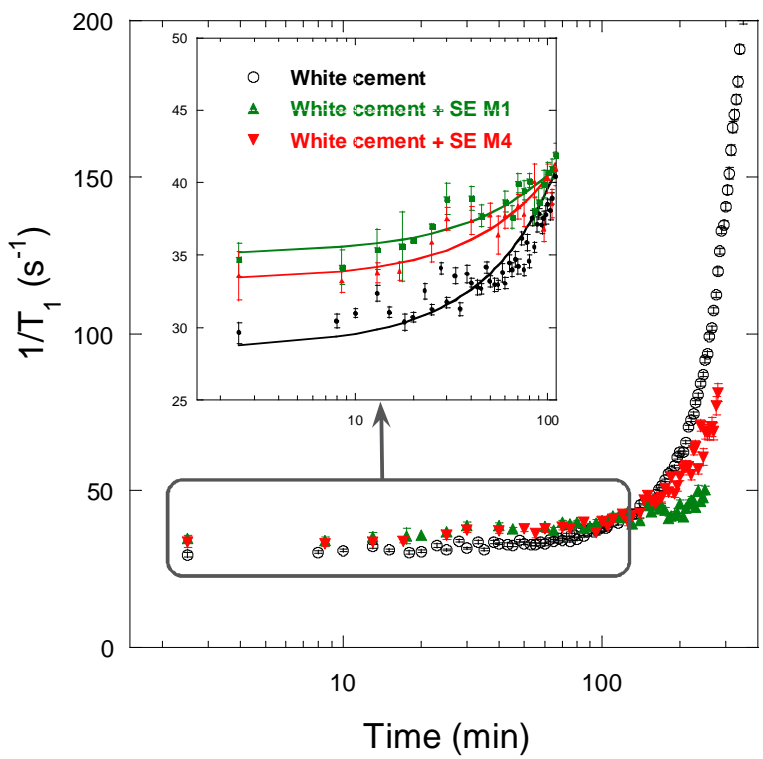

547

548

549

Fig. 7

550 


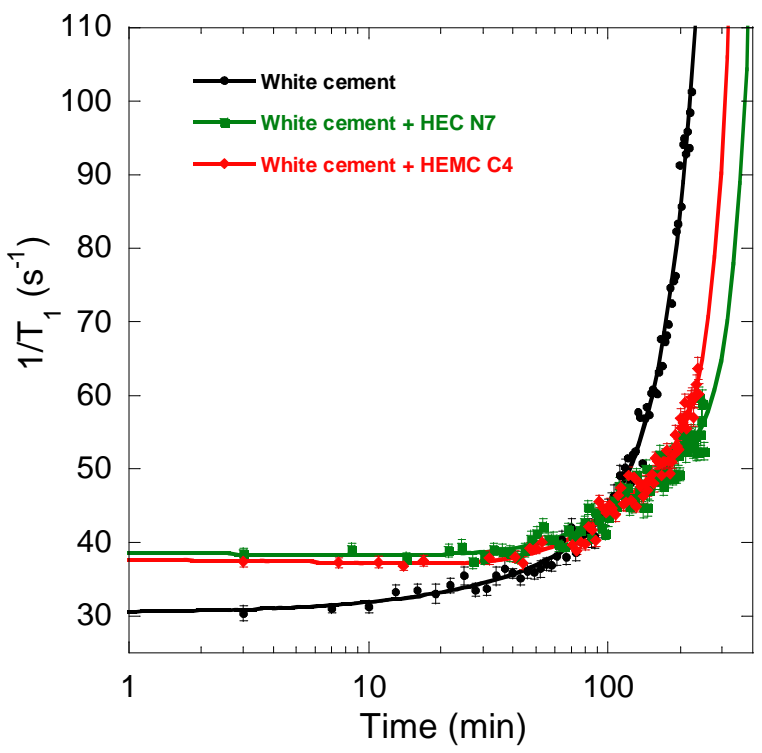

552

553

Fig. 8

554 


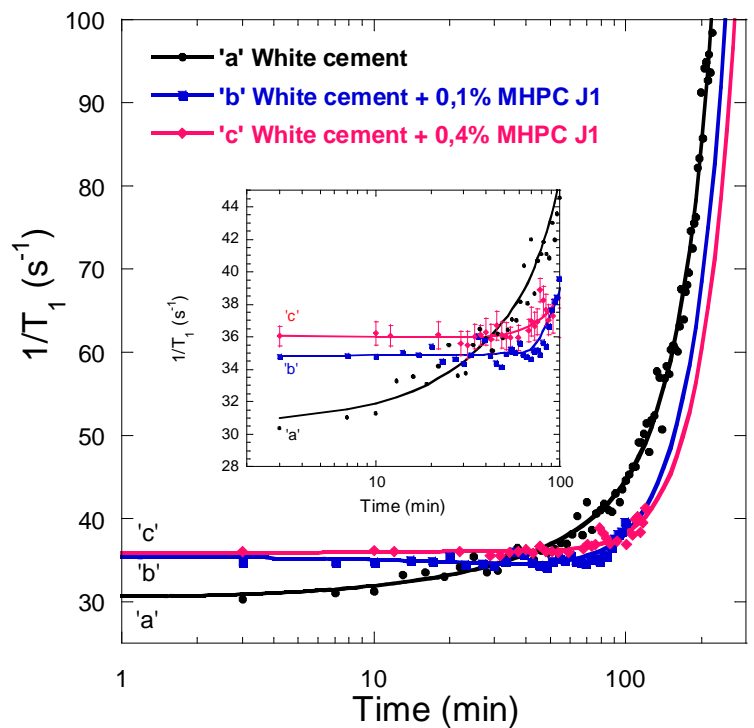

Fig. 9 


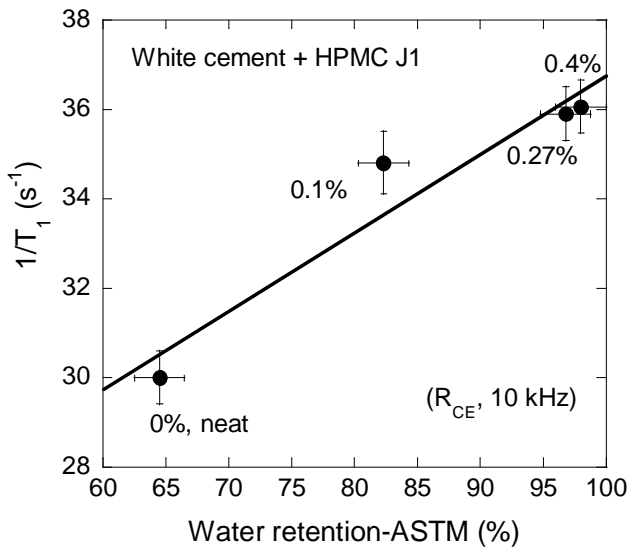

Fig. 10

560 